\title{
A Comparison of The Effects of Lidocaine and Saline Injection on Pain, Disability, and Shear-Wave Elastography Findings in Patients with Myofascial Trigger Points
}

\author{
Pınar Doruk Analan' (1), Hülya Aslan² (D, Sermin Tok Umay 3 (1) \\ 'Department of Physical Medicine and Rehabilitation, Bașkent University School of Medicine, Adana, Turkey \\ 2Department of Radiology, Bașkent University School of Medicine, Adana, Turkey \\ ${ }^{3}$ Department of Radiology, Kütahya Health Sciences University, Kütahya, Turkey
}

ORCID IDs of the authors: P.D.A. 0000-0002-3528-37I2; H.A. 0000-0002-7I38-246X; S.T.U. 0000-0002-716I-016X.

Cite this article as: Doruk Analan P, Aslan H, Tok Umay S. A Comparison of The Effects of Lidocaine and Saline Injection on Pain, Disability, and Shear-Wave Elastography Findings in Patients with Myofascial Trigger Points. Cyprus J Med Sci 2019; 4(2): 103-9.

\begin{abstract}
BACKGROUND/AIMS
To compare the effects of lidocaine injection ( $\mathrm{LI}$ ) and saline injection (SI) on the myofascial trigger points (MTrPs) in the trapezius muscle on pain, disability, and shear-wave elastography (SWE) in patients with myofascial pain syndrome (MPS). The secondary aim was to evaluate the correlations between SWE and pain with disability scores.
\end{abstract}

\section{MATERIAL and METHODS}

This prospective study included 45 patients with MTrPs due to MPS. The patients were evaluated using the visual analog scale (VAS), Neck Disability Index (NDI), and SWE immediately before and I5 days after the injections. The patients were randomly assigned to an $\mathrm{LI}$ ( $n=20,30$ MTrPs) or an SI ( $n=25,32$ MTrPs) group. The LI group was treated with lidocaine, and the SI group was treated with SI.

\section{RESULTS}

Visual analog scale and NDI scores improved significantly in both groups after injection ( $p \leq 0.05)$. In addition, $16 \mathrm{MTrPs}$ in the LI group and $3 \mathrm{MTrPs}$ in the SI group were completely resolved. Maximum shear-wave velocity $(\mathrm{V}(\mathrm{s}) \mathrm{max})$ and mean shear-wave velocity significantly decreased in the SI group after injection $(p=0.025)$. The size of MTrPs decreased in the LI group $(p=0.02)$. Pre-injection $V(s)$ max and resting VAS were weakly correlated $(r=0.309)$. No significant correlation was found on other SWE measurements with VAS and NDI scores $(r<0.3)$.

\section{CONCLUSION}

Lidocaine injection and SI effectively improved the disability and pain in patients with MPS. LI is more effective than SI in reducing the size of the trigger points and resolving MTrPs. SWE findings may not completely reflect the severity of pain and correlate with disability.

Keywords: Disability, pain, lidocaine injection, myofascial trigger points, shear-wave elastography

\section{INTRODUCTION}

Myofascial pain syndrome (MPS) is a syndrome characterized by pain and accompanying muscle spasm, referring pain patterns, stiffness, restricted range of motion caused by myofascial trigger points (MTrPs) on constricted fibers of muscles, and/or fasciae (I). The prevalence of MPS varies from 21\% to 30\% (2). Detecting tenderness, taut bands, and MTrPs depends on the examiner's clinical skills (3). MPS treatment targets breaking down the chain reaction of "spasm-painspasm" and resolving MTrPs. Currently, different modalities, such as MTrPs injections, spray and stretch technique, or physiotherapy, can be used for treatment. These modalities inactivate the MTrPs with various effects (I). Lidocaine hydrochloride is a local anesthetic commonly used for treating pain due to MTrPs. It is a reversible blocker of conduction along the small nerve fibers carrying pain and autonomic impulses $(4,5)$.

Objective characterization and quantitative measurement of the properties of MTrPs can improve their localization, diagnosis, and treatment. Sonographic techniques can play a role in objectively identifying active MTrPs and detecting 
the improvement in outcome measurements after therapeutic interventions (6). Ultrasound (US) elastography is a new, lowcost, fast, and non-invasive technique assessing the stiffness of the lesions in real time. Several elastography techniques, including strain elastography and shear-wave elastography (SWE), are available (7-9). SWE is an objective method and uses shear waves produced by the interaction of the conventional US waves within the tissue (10). It allows direct assessment of elasticity and quantification of the soft tissue stiffness in real time. However, a few studies in the literature have evaluated MTrPs by SWE. This novel study compared lidocaine injection (LI) and saline injection (SI) treatment modalities by SWE.

The primary objective of the present study was to compare the improvement in pain, disability, and SWE measurements with LI and SI into the trapezius muscle with MTrPs. The secondary objective was to evaluate the correlations of SWE measurements with pain and disability scores.

\section{MATERIAL and METHODS}

This prospective, randomized, controlled study was approved by the Baskent University Institutional Review Board and Ethics Committee (Project No.: KAl6/179) and supported by the Baskent University Research Fund. Written informed consent was obtained from all of the patients.

Power analysis during the biostatistical preliminary assessment indicated a study population of 40 patients (20 patients for each group) with 95\% confidence level and 80\%power. Power analysis was performed using mean values of pain intensity according to the study by Ballyns et al. (6). All patients had active MTrPs on the upper part of the trapezius muscle on physical examination. The diagnostic criteria by Simons and Travell were used for diagnosing MPS (II). Simons and Travell's criteria take into account the existence of MTrPs during muscle palpation and restriction in compromised muscles.

Exclusion criteria were as follows: mental retardation, local anesthetic allergy, bleeding diathesis, cervical and/or thoracic disk herniation, radiculopathy, and receiving any drug-targeting pain (e.g., nonsteroidal anti-inflammatory drugs (NSAIDs), paracetamol, and myorelaxant drugs). Patients with injection treatment, manual therapy, massage, or any interventions into the MTrPs within the last 2 months were also excluded from the study.

A total of 66 patients with MPS who were admitted to the physical medicine and rehabilitation outpatient clinic with neck and/ or back pain were enrolled in the study. A physiatrist diagnosed the patients enrolled in the study (researcher I). A flowchart of the study population is shown in Figure I. Eight patients were excluded from the present study for some reasons. The remaining 58 patients were allocated to two groups as LI group and SI group. The patients in the LI group were treated with $0.5 \mathrm{~mL}$ lidocaine without epinephrine $(5 \mathrm{mg} / \mathrm{mL})$, and the patients in the SI group were treated with $0.5 \mathrm{~mL}$ saline $(0.9 \% \mathrm{NaCl})$ injection. A radiologist blinded to the nature of the study selected the patients by drawing lots and randomly assigned 29 patients to each group (researcher 2). Four patients in the SI group and nine patients in the LI group were excluded from the study for reasons including receiving steroid injections into the MTrPs, could not be reached for control evaluation, performing massage or manual therapy into the MTrPs, or using NSAIDs.

Finally, 20 patients (30 MTrPs) in the LI group and 25 patients ( 32 MTrPs) in the SI group were included in the study. The radiologist performing SWE and measuring outcomes (visual analog scale (VAS) and Neck Disability Index (NDI)) was unaware of the groups (researcher 2). In addition, data collection was performed by another radiologist blind to the groups (researcher 3 ).

\section{Outcomes}

\section{Visual analog scale}

The severity of pain during rest, night, and physical activity was assessed by VAS. VAS is a scale evaluating subjective pain intensity from 0 to 10. Studies have shown that VAS is a reliable and valuable method for evaluating $\operatorname{MTrPs}(12,13)$.

\section{Neck disability index}

Neck Disability Index was designed for assessing neck pain and disability. It contains 10 self-reported items, including pain intensity, personal care, lifting, reading, headache, concentration, work, driving, sleeping, and recreation. Each item is scored on a 6-point scale from 0 (no disability) to 5 (full disability). The sum score of all 10 items is calculated using a percentage of the maximal score, with higher values representing greater disability (14). The total score ranges from 0 (no disability) to 50 (total disability) or, in percentage terms, between 0 and 100. The modified Turkish version of NDI was used in the present study. Kesiktas et al. (12) demonstrated that this version is a reliable and valid test suitable for daily practice.

\section{Shear-wave elastography}

Shear-wave elastography was performed by a blind radiologist experienced in musculoskeletal imaging. The US elastography evaluations were performed using a US system (Acuson S2000; Siemens, Erlangen, Germany) with a linear transducer that enabled scanning with a frequency ranging from 4 to $9 \mathrm{MHz}$. Elastography images were obtained using a freehand technique at the same time as US. US elastography was performed when the patient was in a prone position with arms resting alongside their trunk. The transducer was held in the transverse plane over the trapezius muscle. Sikdar et al. (I3) defined MTrPs as focal hypoechoic areas with heterogeneous echotexture on B-mode US. Their criteria were used, but both latent and active MTrPs might have the same imaging characteristics. Thus, the lesion was gently palpated to confirm tenderness and referring pain. B-mode US and SWE were performed for each lesion. The localization of the active MTrPs was marked on the skin before injection. The marked areas were photographed for assessing the same areas 15 days after injection. Two to four regions of interest (ROIs) were calculated due to the size of the lesions (Figure 2). In addition, $1.5 \times 1.5 \mathrm{~mm}^{2}$ box-shaped standard ROls automatically provided by the US system were used. Application of high pressure was avoided. The qualities of the images were assessed by color-coded quality maps provided by the US system. The color-coded green areas in the maps were considered reliable, whereas yellow and red color-coded areas were considered low-quality scans. All scans were repeated at least three times in MTrPs, and the best representative image with the highest quality on the quality map was selected. Qualitative 


\section{Flowchart of the Study Population}
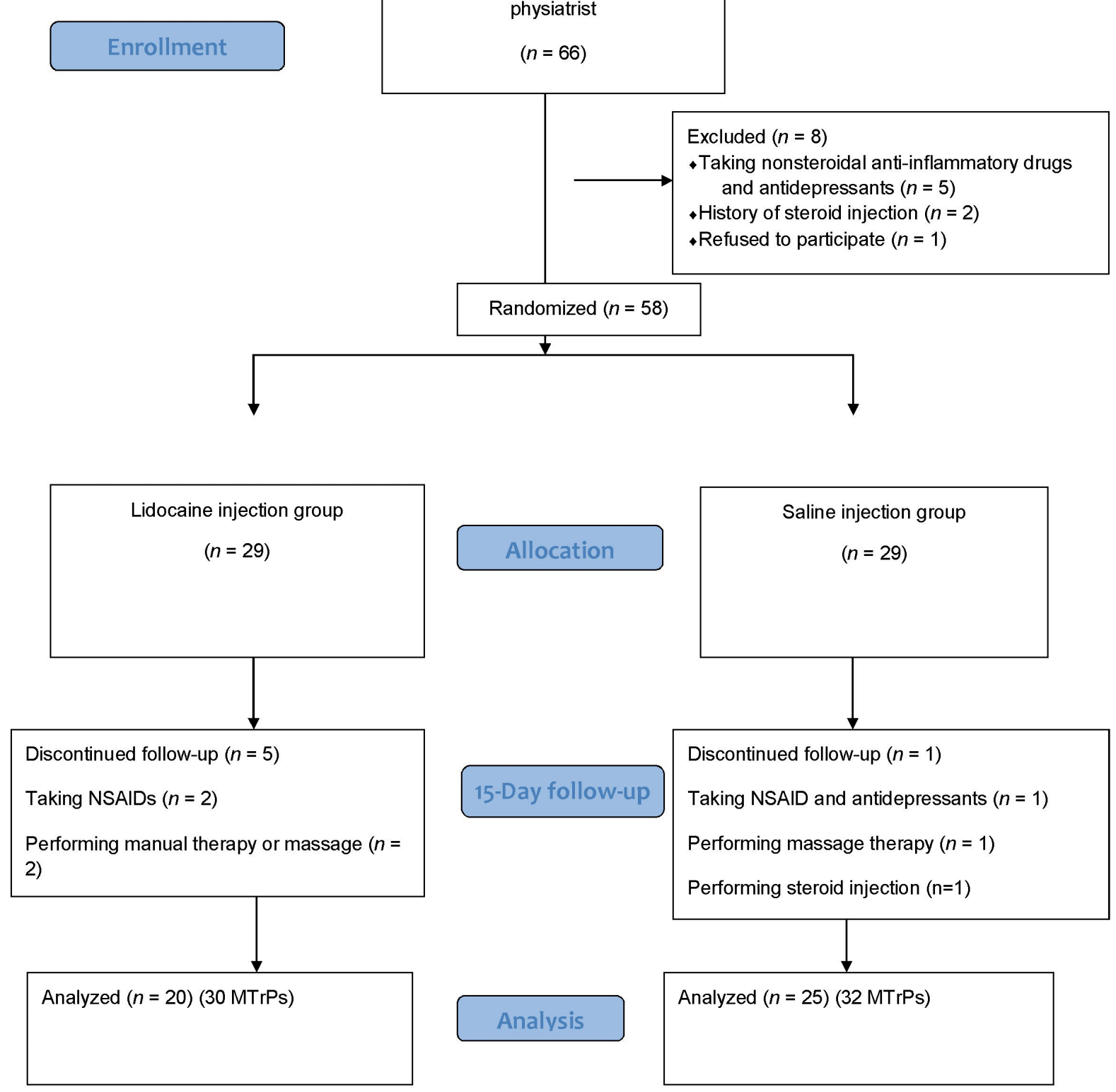

FIGURE I. Flowchart of the study population

color-coded elastography images and quantitative maps measuring shear-wave velocity were obtained. Mean and maximum shear-wave velocities ( $\mathrm{V}(\mathrm{s})$ mean and $\mathrm{V}(\mathrm{s})$ max) were assessed. All of the images were evaluated at a workstation (Synapse version 4.0; Fujifilm Medical Systems Inc., CT, USA).

\section{Injections}

Baseline SWE was performed before the injection by a radiologist who was blind to the groups. The MTrPs were marked on the skin, and the depth of the lesion was noted by the performing radiologist. US guidance was used for injection into the trigger point. In the LI group, $0.5 \mathrm{~mL}$ of $0.5 \%$ lidocaine hydrochloride without epinephrine $(5 \mathrm{mg} / \mathrm{mL}$ ) was injected into the MTrPs by a single physiatrist. Antisepsis was performed using 10\% polyvinylpyrrolidone iodine (Batticonol, DERMOSEPT), and a sterile needle with a thickness of 26 gauge $\times 1 / 2\left(0.45 \times 13 \mathrm{~mm}^{2}\right)$ was inserted into the MTrPs at an angle of $30^{\circ}$ with respect to the skin. The needle was aspirated before injecting a small amount of injection solution to ensure that the needle was not in a blood vessel. Then, the needle was withdrawn to the subcutaneous tissue into the MTrPs. In the SI group, $0.5 \mathrm{~mL}$ saline $(0.9 \% \mathrm{NaCl})$ was injected to the MTrPs in the same way. The same physiatrist (researcher I) prepared the injection materials and injected them into the MTrPs of the patients. The patients in both the groups were blind to the SI or LI materials.

The patients were instructed not to use any drugs or interventions targeting pain or preventing other symptoms during the study. All of the patients were re-evaluated 15 days after the intervention. 


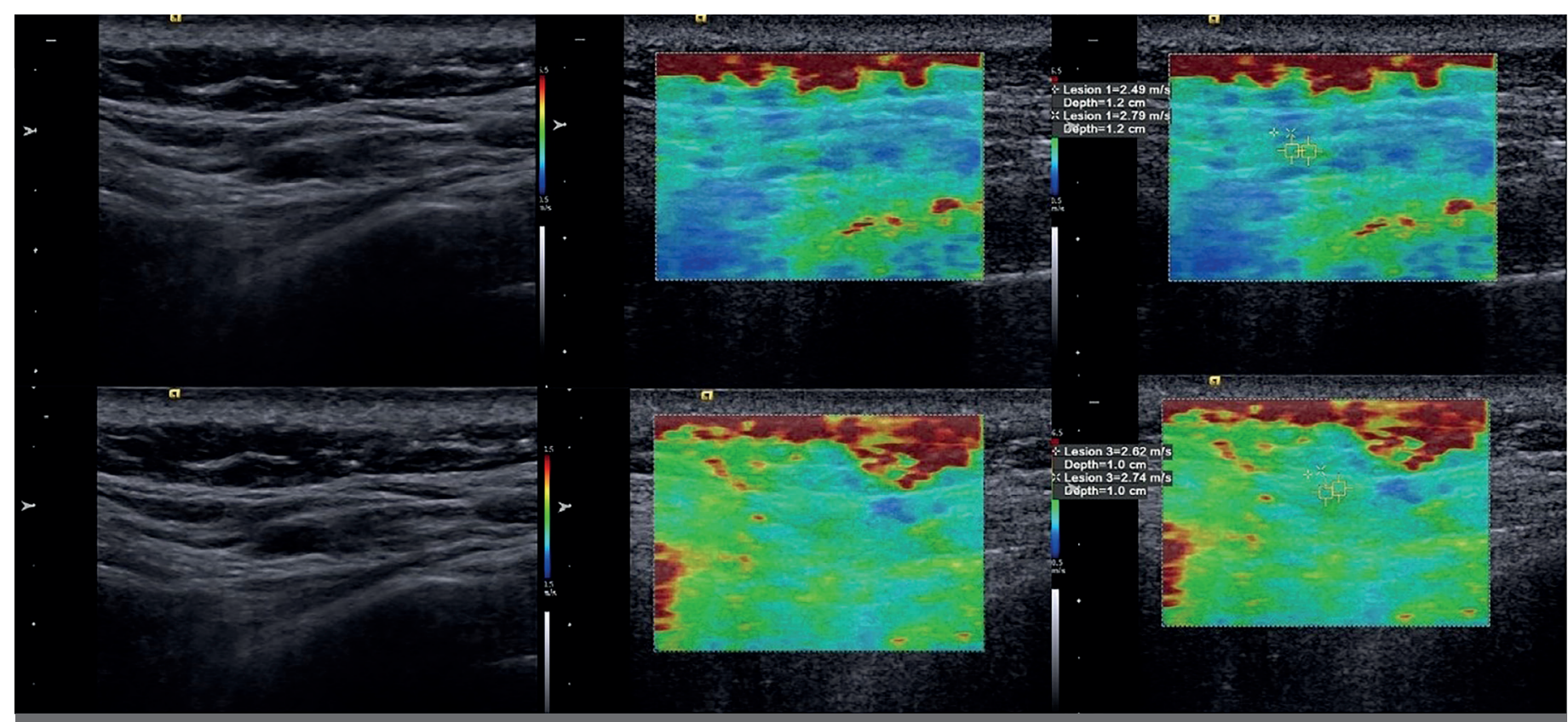

FIGURE 2. A 35-year-old man with active MTrP within the trapezius muscle. Upper row demonstrates pretreatment US and SWE images. Lower row shows US and SWE images after saline injection treatment

\section{Data and Statistical Analysis}

Statistical analysis was performed using the Statistical Package for the Social Sciences software version 17.0 (SPSS Inc., Chicago, IL, USA). If continuous variables were normal, they were described as mean \pm standard deviation ( $p>0.05$ in Kolmogorov-Smirnov or Shapira-Wilk test $(n<30)$ ); and if they were non-normal, they were described as median. The Student's $t$ test was used for comparisons between the groups with normally distributed data, and the Mann-Whitney Utest was used for comparisons between the groups with non-normally distributed data. The Wilcoxon rank-sum test was used to analyze pre- and post-intervention measures. Pearson's chi-square and Fisher's exact tests were used for comparison of categorical variables. A p value $\leq 0.05$ was considered statistically significant.

Correlations were tested using the Pearson's correlation test, and the correlation coefficients were interpreted as either excellent $(r \geq 0.91)$, good $(0.90 \geq r \geq 0.71)$, fair $(0.70 \geq r \geq 0.51)$, weak $(0.50 \geq r \geq 0.31)$, or little or none $(r \leq 0.3)$. A p value $\leq 0.05$ was considered statistically significant. Type I and 2 error risks were kept at the minimum level by applying the appropriate power analysis during the biostatistical preliminary assessment stage.

\section{RESULTS}

The LI and SI groups included 30 and $32 \mathrm{MTrPs}$, respectively. The mean age, gender, and affected dominant extremities did not show statistically significant difference between the groups ( $p>0.05$ ) (Table l). When we compared the patients who attended and who did not attend the treatment in each group, the result is not statistically significant $(p>0.05)$.

Pre-intervention values of the VAS and NDI and the SWE measurements were similar for both the groups. The only exception was pretreatment size of the MTrPs. It was found to be higher in the LI group $(p=0.02)$. VAS and NDI scores were significantly improved with both $\mathrm{LI}$ and SI injections $(\mathrm{p} \leq 0.05)$.

\begin{tabular}{|c|c|c|c|}
\hline Characteristics & $\begin{array}{l}\text { Group I } \\
(n: 20)\end{array}$ & $\begin{array}{l}\text { Group } 2 \\
\text { (n: 25) }\end{array}$ & $\mathbf{p}$ \\
\hline Age (years) (mean $\pm S D)$ & $45.6 \pm 13.22$ & $42.4 \pm 11.16$ & 0.783 \\
\hline $\begin{array}{l}\text { Gender } \\
\text { (Females/Males) (\%) }\end{array}$ & $13 / 7(65 / 35)$ & $19 / 6(76 / 24)$ & 0.604 \\
\hline $\begin{array}{l}\text { Dominant extremity } \\
\text { (right/left) }(\%)\end{array}$ & $18 / 2(90 / 10)$ & $24 / I(96 / 4)$ & 0.415 \\
\hline $\begin{array}{l}\text { Affected extremity } \\
\text { (right/left/bilateral) (\%) }\end{array}$ & $7 / 7 / 6(35 / 35 / 30)$ & $8 / 14 / 3(32 / 56 / 12)$ & 0.237 \\
\hline
\end{tabular}

A comparison of the pre- and post-injection SWE showed that $(\mathrm{s})$ max and $(\mathrm{s})$ mean significantly decreased in the SI group $(p=0.022$ and $p=0.025$, respectively), but they did not change significantly in the LI group ( $p \geq 0.05$ ). In addition, 14 of MTrPs in the LI group and 3 of the MTrPs in the SI group were completely resolved after injection. One of the MTrPs was very superficial and did not provide the distance to place the box of ROI after treatment in the SI group.

A comparison of the post-intervention outcome parameters revealed that VAS, NDI, and all of the SWE measurements of the post-treatment period were similar for the groups ( $p>0.05)$. Preand post-treatment VAS and NDI results are shown in Table 2. B-mode US and SWE results are summarized in Table 3. In addition, $(\mathrm{s}$ ) max and resting VAS were found to be weakly correlated on evaluating the correlation of VAS and NDI with $\mathrm{K}(\mathrm{s})$ max and $\mathrm{K} s$ )mean ( $r=0.309$ ). No significant correlation of other SWE measurements with VAS and NDI scores was found $(r<0.3$, $p>0.05$ ). There were no serious side effects following injections including prolonged bleeding and signs of an allergic reaction, such as difficulty breathing or facial swelling. Two patients had moderate pain at the injection side, and one patient had redness, swelling, or warmth at the injection site without swelling or pain. 


\begin{tabular}{|c|c|c|c|}
\hline Characteristics & Group I & Group 2 & $p$ \\
\hline \multicolumn{4}{|c|}{ Neck Disability Index (\%) } \\
\hline Before treatment & $28.3 \pm 11.9$ & $27.36 \pm 17.97$ & 0.842 \\
\hline After treatment & $16.3 \pm 16.26$ & $21.5 \pm 18.93$ & 0.339 \\
\hline$p^{*}$ & 0.001 & 0.022 & \\
\hline \multicolumn{4}{|l|}{ VAS/rest } \\
\hline Before treatment & $6.3 \pm 2.81$ & $6.12 \pm 2.11$ & 0.807 \\
\hline After treatment & $4.5 \pm 3.41$ & $4.44 \pm 2.97$ & 0.950 \\
\hline$p^{*}$ & 0.043 & 0.004 & \\
\hline \multicolumn{4}{|l|}{ VAS/night } \\
\hline Before treatment & $6.65 \pm 3.32$ & $6.84 \pm 3.11$ & 0.845 \\
\hline After treatment & $4.4 \pm 3.79$ & $4.88 \pm 4.05$ & 0.687 \\
\hline$p^{*}$ & 0.013 & 0.009 & \\
\hline \multicolumn{4}{|c|}{ VAS/physical activity } \\
\hline Before treatment & $6.4 \pm 3.31$ & $6.32 \pm 3.65$ & 0.940 \\
\hline After treatment & $4.5 \pm 3.12$ & $4.48 \pm 3.51$ & 0.984 \\
\hline$p^{*}$ & 0.013 & 0.010 & \\
\hline
\end{tabular}

\begin{tabular}{|c|c|c|c|}
\hline Characteristics & Group I & Group 2 & $\mathrm{p}$ \\
\hline \multicolumn{4}{|l|}{$\mathrm{SW} V$ mean $(\mathrm{m} / \mathrm{s})$} \\
\hline Before treatment & $3.17 \pm 0.81$ & $3.66 \pm 1.56$ & 0.131 \\
\hline After treatment & $3.22 \pm 1.33$ & $2.91 \pm 0.84$ & 0.338 \\
\hline$p^{*}$ & 0.981 & 0.022 & \\
\hline \multicolumn{4}{|l|}{$\operatorname{SWVmax}(\mathrm{m} / \mathrm{s})$} \\
\hline Before treatment & $3.29 \pm 0.92$ & $3.82 \pm 1.64$ & 0.123 \\
\hline After treatment & $3.27 \pm 1.32$ & $3.07 \pm 0.95$ & 0.548 \\
\hline$p^{*}$ & 0.913 & 0.025 & \\
\hline \multicolumn{4}{|l|}{ Size of MTrPs (mm) } \\
\hline Before treatment & $7.79 \pm 2.78$ & $5.65 \pm 2.44$ & 0.002 \\
\hline After treatment & $5.61 \pm 2.26$ & $5.11 \pm 3.49$ & 0.59 \\
\hline$p^{*}$ & 0.002 & 0.13 & \\
\hline \multicolumn{4}{|c|}{$\begin{array}{l}\text { p*: Wilcoxon signed-rank test } \\
\text { SWV: shear-wave velocity; SWVmax: maximum shear-wave velocity; } \\
\text { Group I: lidocaine injection+dry needling; Group 2: saline injection+dry } \\
\text { needling }\end{array}$} \\
\hline
\end{tabular}

\section{DISCUSSION}

The aim of the present study was to compare the effects of LI and $\mathrm{SI}$ injections into the MTrPs in the trapezius muscle on pain, disability, and SWE findings in patients with MPS. The secondary aim was to evaluate the correlations between SWE results, pain, and disability scores. Pain and disability due to MTrPs were improved with both SI and LI treatments. However, LI did not appear to be more effective than $\mathrm{SI}$ in improving pain and disability. Based on these data, it was thought that patients with
MTrPs could be treated with lidocaine for improving pain and disability. Lugo et al. (2) investigated LI and physiotherapy, alone or in combination, in patients with MTrPs. They found no difference in pain and quality of life between these treatments.

Although the exact etiology of MTrPs is unknown, one theory is that chronic muscle overuse leads to inflammation (I5). Moreover, histological changes within the muscle in response to pain may correspond to changes in mechanical properties. Ballyns et al. (16) showed that $M(s)$ is significantly higher in active MTrPs and the surrounding tissue than in the normal tissue. Clinical application of elastography may provide an objective assessment for identifying MTrPs and detecting their changes (15-18). US elastography is a useful tool for monitoring response to injections into trigger points (19). The present study found that pretreatment sizes of the MTrPs in the LI group were significantly higher, but they significantly reduced after treatment. However, the size of MTrPs did not reduce significantly in the SI group after treatment. After injection, more MTrPs in the LI group than those in the SI group were completely resolved. Based on these data, it is proposed that $\mathrm{LI}$ injection may be a good option in the local treatment of MTrPs. On the contrary, $M(s)$ significantly improved only in the SI group in the present study.

Despite the application of SWE to healthy human skeletal muscles, its use in assessing the treatment of MTrPs with injections has not been investigated adequately. This novel study compared the effects of $\mathrm{LI}$ and SI treatment modalities using SWE. Maher et al. (14) evaluated the effects of dry needling and posture on MTrPs using SWE. Seven women with palpable MTrPs in the upper trapezius muscle were investigated in the study. They showed a reduction in the shear modulus after dry needling and a significant difference between prone and upright positions while sitting. They proposed that SWE detected the changes in MTrPs (14). The present study found that some SWE measurements (presence of MTrPs, size of MTrPs, $(s)$ mean, and $(\mathrm{s}$ ) max) changed with injections. Thus, the results agreed with the findings of Maher et al. (I4) on the feasibility of SWE for detecting the changes in MTrPs.

Müller et al. (20) designed a double-blind, randomized, controlled, pilot study for evaluating the effects of acupuncture (AC) and electroacupuncture (EA) in women with painful MTrPs in the upper trapezius muscle using two-dimensional US and US elastography. They found that EA treatment decreased general and local pain intensity, whereas only general pain was decreased in the AC group (20). The post-treatment strain ratios did not change between the groups.

Moreover, a weak correlation was found between pretreatment (s)max and resting VAS. Other outcome measurements were not correlated with each other, although the size of MTrPs is a very good classifier of the site type in the upper trapezius muscle and also quick and simple to implement using clinical sonography. Ballyns et al. (16) did not find a correlation between trigger point size and pain pressure threshold score. They suggested that other mechanisms could be contributing independently to the trigger point size and pain sensitivity (16). No correlation was found between MTrPs size and VAS scores, consistent with the present results. In addition, it was thought that SWE measurements (except $(\mathrm{s}$ ) max) could be affected by more complicated mechanisms beyond functional outcomes. 
Pretreatment VAS, NDI, and SWE measurements were used for correlation analysis because injection treatments could affect these outcomes. Some patients had multiple MTrPs in the present study. When the VAS and NDI are used once in patients with multiple MTrPs, it is difficult to differentiate which trigger point is being reflected. This limitation might have affected the present correlation analysis. In addition, there is another technical difficulty that must be mentioned. MTrP in one patient was very superficial and did not provide the distance to place the box of ROI to measure $\mathrm{Ms}$ ). The use of gel pads would be better in such conditions (2I). SWE provides a quantitative measure of the lesion stiffness obtained by either in a small fixed ROI (single measurement) or pixel-by-pixel in a field-of-view box giving a color map (22). Tissue stiffness measurements in an ROI can be displayed in speed $(\mathrm{m} / \mathrm{s}$ ) or in pressure/elasticity $(\mathrm{kPa})$ depending on the commercially available different US units. As the velocity increases, it suggests increased tissue stiffness.

The first limitation of the present study was that pain was scored by VAS, which is a patient-dependent subjective method. Compared with VAS, algometers are more objective tools for evaluating pain intensity. Algometric evaluation methods are designed to measure deep pressure pain thresholds or tenderness resistance. Hence, algometers can be a more useful option for assessing the treatment effects in these cases (23). The outpatient clinic involved in the present study did not have an algometer. Therefore, the only option was to assess the pain intensity by VAS. Another limitation of the study is that all the elastographic measurements were performed by a single radiologist. It would be better to assess the intrarater reliability of the elastography. Other limitations of the present study included the short duration of the follow-up period and the limited number of patients. Further studies with larger sample sizes and longer follow-up periods are required to independently confirm the present findings. However, the study included patients with multiple MTrPs. Therefore, VAS and NDI results were not completely correlated with SWE measurements. SWE directly measures each MTrP separately; however, VAS and NDI generally evaluate pain regardless of the number of MTrPs. When VAS and NDI are used once in patients with multiple MTrPs, it is difficult to differentiate which trigger point is being reflected. Algometers can be more specific tools in patients with multiple MTrPs and can specifically evaluate each MTrPs. Thus, algometer and SWE measurement correlations can be good evaluation methods for correlation analysis in further studies. Evaluation of patients with single MTrPs can be a second option. In addition, adding another group with just dry needling should be better to compare the effectiveness of these two treatment modalities.

In conclusion, $\mathrm{LI}$ is a useful option for short-term treatment of patients with MPS with neck and/or back pain due to the MTrPs in the trapezius muscle. It can be used to reduce pain, disability, and trigger point size and to resolve trigger points. LI and SI effectively improve pain and disability in MPS. SWE can be used for assessing the size of these painful trigger points. However, SWE measurements may not completely reflect the severity of pain and disability in patients with MTrPs due to MPS.

Ethics Committee Approval: Ethics committee approval was received for this study from Baskent University Institutional Review Board and Ethics Committee (Approval Date: 16.06.2016, Approval Number: 16/66).
Informed Consent: Informed consent was obtained from the patients who participated in this study.

Peer-review: Externally peer-reviewed.

Author contributions: Concept - S.T.U., P.D.A., H.A.; Design - S.T.U., P.D.A.; Supervision - S.T.U.; Resource - P.D.A., H.A.; Materials - S.T.U., P.D.A., H.A.; Data Collection and/or Processing - S.T.U., H.A.; Analysis and/or Interpretation - P.D.A., H.A.; Literature Search - P.D.A., H.A.; Writing - S.T.U., P.D.A., H.A.; Critical Reviews - H.A., S.T.U.

Acknowledgements: The authors would like to thank Çağla Sarıtürk for the statistical analyzes and interpretion of the data.

Conflict of Interest: The authors have no conflicts of interest to declare.

Financial Disclosure: The authors declared that this study has received no financial support.

\section{REFERENCES}

I. Kavadar G, Çağlar N, Özen Ș, Tütün Ș, Demircioğlu D. Efficacy of conventional ultrasound therapy on myofascial pain syndrome: a placebo controlled study. Agr 2015; 27: 190-6. [CrossRef]

2. Lugo LH, García HI, Rogers HL, Plata JA. Treatment of myofascial pain syndrome with lidocaine injection and physical therapy, alone or in combination: a single blind, randomized, controlled clinical trial. BMC Musculoskelet Disord 2016; 17: 10I. [CrossRef]

3. Park GY, Kwon DR. Application of real-time sonoelastography in musculoskeletal diseases related to physical medicine and rehabilitation. Am J Phys Med Rehabil 2011; 90: 875-86. [CrossRef]

4. Hsu WC, Wang TL, Lin YJ, Hsieh LF, Tsai CM, Huang KH. Addition of lidocaine injection immediately before physiotherapy for frozen shoulder: a randomized controlled trial. PLoS One 2015; 10: e0118217. [CrossRef]

5. Alvarez DJ, Rockwell PG. Trigger points: diagnosis and management. Am Fam Physician 2002; 65: 653-60.

6. Ballyns JJ, Turo D, Otto P, Shah JP, Hammond J, Gebreab T, et al. Office-based elastographic technique for quantifying mechanical properties of skeletal muscle. J Ultrasound Med 2012; 31: 1209-19. [CrossRef]

7. Drakonaki EE, Allen GM, Wilson DJ. Ultrasound elastography for musculoskeletal applications. Br J Radiol 2012; 85: 1435-45. [CrossRef]

8. Garra BS. Imaging and estimation of tissue elasticity by ultrasound Ultrasound Q 2007; 23: 255-68. [CrossRef]

9. Garra BS. Elastography: current status, future prospects, and making it work for you. Ultrasound Q 2011; 27: 177-86. [CrossRef]

10. Bercoff J, Tanter M, Fink M. Supersonic shear imaging: a new technique for soft tissue elasticity mapping. IEEE Trans Ultrason Ferroelectr Freq Control 2004; 51: 396-409. [CrossRef]

II. Simons DG, Travell J, Simons L. Myofascial pain and dysfunction: The trigger point manual. 2nd ed., Vol I. Baltimore, MD: William $\bar{\alpha}$ Wilkins; 1999.

12. Kesiktas N, Ozcan E, Vernon H. Clinimetric properties of the Turkish translation of a modified neck disability index. BMC Musculoskelet Disord 2012; 13: 25. [CrossRef]

13. Sikdar S, Shah JP, Gebreab T, Yen RH, Gilliams E, Danoff J, et al. Novel applications of ultrasound technology to visualize and characterize myofascial trigger points and surrounding soft tissue. Arch Phys Med Rehabil 2009; 90: 1829-38. [CrossRef]

14. Maher RM, Hayes DM, Shinohara M. Quantification of dry needling and posture effects on myofascial trigger points using ultrasound shear-wave elastography. Arch Phys Med Rehabil 2013; 94: 2146-50. [CrossRef]

15. Bron C, Dommerholt JD. Etiology of myofascial trigger points.Curr Pain Headache Rep 2012; 16: 439-44. [CrossRef]

16. Ballyns JJ, Turo D, Otto P, Shah JP, Hammond J, Gebreab T, et al. Office-based elastographic technique for quantifying mechanical properties of skeletal muscle. J Ultrasound Med 2012; 31: 1209-19. [CrossRef] 
17. Chan ST, Fung PK, Ng NY, Ngan TL, Chong MY, Tang CN, et al. Dynamic changes of elasticity, cross-sectional area, and fat infiltration of multifidus at different postures in men with chronic low back pain. Spine J 2012; 12: 38I-8. [CrossRef]

18. Kuo WH, Jian DW, Wang TG, Wang YC. Neck muscle stiffness quantified by sonoelastography is correlated with body mass index and chronic neck pain symptoms. Ultrasound Med Bio 2013; 39: 1356-61. [CrossRef]

19. Brandenburg JE, Eby SF, Song P, Zhao H, Brault JS, et al. Ultrasound elastography: the new frontier in direct measurement of muscle stiffness.Arch Phys Med Rehabil 2014; 95: 2207-19. [CrossRef]

20. Müller CE, Aranha MF, Gavião MB. Two-dimensional ultrasound and ultrasound elastography imaging of trigger points in women with myofascial pain syndrome treated by acupuncture and elec- troacupuncture: a double-blinded randomized controlled pilot study. Ultrason Imaging 2015; 37: 152-67. [CrossRef]

21. De Zordo T, Fink C, Feuchtner GM, Smekal V, Reindl M, Klauser AS Real-time sonoelastography findings in healthy Achilles tendons. AJR Am J Roentgenol 2009; 193: WI34-8. [CrossRef]

22. Barr RG, Nakashima K, Amy D, Cosgrove D, Farrokh A, Schafer F, et al. WFUMB guidelines and recommendations for clinical use of ultrasound elastography: Part 2: breast. Ultrasound Med Biol 2015; 41: II26-47. [CrossRef]

23. Park G, Kim CW, Park SB, Kim MJ, Jang SH. Reliability and usefulness of the pressure pain threshold measurement in patients with myofascial pain. Ann Rehabil Med 2011; 35: 412-7. [CrossRef] 